\title{
Evidence of Inhaled Tobramycin in Non-Cystic Fibrosis Bronchiectasis
}

\author{
Montserrat Vendrell ${ }^{*}, 1,2$, Gerard Muñoz ${ }^{1}$ and Javier de Gracia ${ }^{2,3}$
}

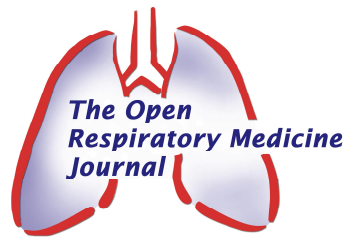

\author{
${ }^{I}$ Bronchiectasis Group. [Girona Biomedical Research Institute] IDIBGI, Dr Trueta University Hospital, Girona, Spain \\ ${ }^{2}$ CIBER de Enfermedades Respiratorias (Ciberes CB06/06/0030), Instituto de Salud Carlos III, Spain \\ ${ }^{3}$ Department of Pulmonology, Hospital Vall d'Hebron, Universitat Autònoma de Barcelona
}

\begin{abstract}
There is currently less experience with inhaled tobramycin in non-cystic fibrosis bronchiectasis than in cystic fibrosis (CF). Intravenous formulation and solution for inhalation (TSI) have been studied in non-CF bronchiectasis patients with chronic $P$. aeruginosa bronchial infection. An improvement in clinical parameters and a reduction in bacterial density have been shown with both inhaled solutions in these patients. However, further trials are needed to determine the most effective dose and administration protocol in these patients. Based on the current evidence, recommendations cannot be made regarding the use of TSI to treat exacerbations. Although no systemic toxicity has been reported in studies specifically investigating this treatment, patients with known kidney disease or ear disorders should be treated with caution. Adverse respiratory effects are reported to be more common in non-CF patients than in CF patients, who tend to be non-smokers and younger. Research is being conducted into the possibility of combining tobramycin with other antibiotics to increase its antibacterial activity. In this review we will present and discuss the published evidence regarding the use of inhaled tobramycin in non-CF bronchiectasis.
\end{abstract}

Keywords: Bronchiectasis, inhaled, tobramycin.

\section{INTRODUCTION}

The aim of antibiotic treatment in acute bronchial infection is to kill and eradicate bacteria. However, in chronic bronchial infection, where bacterial eradication is usually not feasible, the aim of treatment is to reduce the bacterial load and thus reduce the inflammatory response.

Managing patients with bronchiectasis and chronic bronchial infection is a challenge due to the fact that oral antibiotics do not achieve sufficient concentrations at the infection site. Administering inhaled antibiotics to these patients was first suggested in the 1940s [1]. The intention was to release the antibiotic directly into the infected area to achieve high concentrations at this site while reducing systemic side effects. Various intravenous antibiotics, including tobramycin [2-4], have been administered empirically for over 40 years at varying doses and using different nebulisers especially in patients with cystic fibrosis (CF) with chronic Pseudomonas aeruginosa bronchial infection, which is associated with high morbidity and mortality rates. After the study by Ramsey et al., in which patients with CF-related bronchiectasis and chronic $P$. aeruginosa bronchial infection were treated with a specially designed aerosol formulation of tobramycin using a new jet nebuliser at higher doses than had previously been used [5],

*Address correspondence to this author at the Bronchiectasis Group. IDIBGI, Dr Trueta University Hospital, Girona, Avd França s/n ( $9^{\circ}$ planta), 17007 Girona, Spain; Tel: +34 972940294; Fax: +34 972940220;

E-mail: mvendrell.girona.ics@gencat.cat few doubts remain as to the efficacy of this treatment in these patients [6].

Tobramycin is an aminoglycoside with good in vitro antibacterial activity against $P$. aeruginosa, even surpassing gentamicin, and which is also active against other gramnegative (Enterobacteria, Haemophilus influenzae) and some gram-positive aerobic bacteria (Staphilococcus aureus). Its antibacterial effect interferes with the synthesis of bacterial proteins by altering their cell membrane permeability, thereby causing cell death [7]. It is bactericidal at concentrations equal to or slightly higher than inhibitory concentrations and its effect is concentration-dependent under aerobic conditions [8]. In addition to tobramycin's antibacterial effect, it may also inhibit the expression and production of Pseudomonas virulence factors and the cytotoxic effect of neutrophil-derived myeloperoxidase [7]. Like other aminoglycosides, it barely penetrates bronchial secretions when administered parenterally and high doses are required to achieve sufficient concentrations at the infection site, which entails a risk of nephrotoxicity and ototoxicity [7]. Administering aminoglycosides via the inhaled route, higher concentrations can be obtained at the infection site, so reducing systemic absorption and side effects.

A review has been published regarding the evidence of inhaled tobramycin in patients with CF [9]. However, to the best of our knowledge, no review has been made of evidence from patients with non-CF bronchiectasis. The aim of this review is therefore to analyse the available evidence of treatment with tobramycin in patients with non-CF bronchiectasis. 


\section{AVAILABLE FORMULATIONS AND NEBULISERS}

Intravenous tobramycin sulphate solutions containing varying amounts of preservatives and bisulphites, which have been related to bronchoconstriction [10], were used for many years until tobramycin solution for inhalation (TSI) came onto the market. TSI is additive-free and specifically designed to be nebulised into the lungs [8].

TSI was approved by the FDA in 1998 for the treatment of chronic $P$. aeruginosa bronchial infection in patients with CF [8]. It contains high doses of tobramycin $(300 \mathrm{mg})$, is non-pyrogenic and preservative-free, has adjusted $\mathrm{pH}$ and osmolality, and is supplied in single-dose vials so avoiding preparation errors. CF trials have demonstrated the efficacy of TSI with the PARI LC PLUS ${ }^{\circledR}$ nebuliser and the DeVilbiss Pulmo-Aide ${ }^{\circledR}$ compressor [5]. However, it has also been found that when other compressors, such as SystAm 23ST, MedicAid CR50 and CR60, PARI MASTER ${ }^{\circledR}$ and PARI BOY ${ }^{\circledR}$, have been used with the PARI LC PLUS ${ }^{\circledR}$ nebuliser, similar doses of tobramycin are delivered [11]. The PARI eFlow ${ }^{\mathbb{B}}$ rapid is an electronic nebuliser that reduces the nebulisation time of TSI to 7 minutes compared with 20 minutes using the PARI LC PLUS ${ }^{\circledR}$ [12]. Initially, only the $5 \mathrm{ml}$ formulation, containing $300 \mathrm{mg}$ of tobramycin, $11.25 \mathrm{mg}$ of sodium chloride, water for injection and sulphuric acid, along with sodium hydroxide to adjust the $\mathrm{pH}$, was available (TOBI ${ }^{\circledR}$ Novartis AG, Basel, Switzerland) although there is now a more concentrated formulation at the same dose in $4 \mathrm{ml}$ of preservative-free saline $\left(\right.$ Bramitob $^{\mathbb{B}}$, Chiesi Farmaceutici S.p.A., Parma, Italy) that reduces the inhalation time [13] and has demonstrated non-inferiority with regards to the $5 \mathrm{ml}$ formulation in CF patients [14]. This latter formulation is efficiently nebulised using the PARI LC PLUS $^{\circledR}$ nebuliser and the PARI TurboBOY ${ }^{\circledR}$ compressor [14].

The dry powder formulation using the T-326 Inhaler is the most recent tobramycin formulation to be released on the market. The safety and efficacy profile of the $112 \mathrm{mg}$ dose of tobramycin inhalation powder (TIP) in 4 capsules every 12 hours in CF patients is comparable to that of TSI with the PARI LC PLUS ${ }^{\mathbb{R}}$ nebuliser, but the administration time is significantly shorter (an average of 5.6 minutes for TIP $v s$ 19.7 minutes for TSI) [15]. This formulation also reduces the potential for device contamination. No trials have so far been published in patients with non-CF bronchiectasis.

A new formulation, T100 (VANTOBRA, $170 \mathrm{mg} / 1.7$; PARI Pharma GmbH, Stanberg, Germany), is being studied in a solution with lower TSI doses and a higher concentration. T100 is administered with a drug-specific efficient eFlow nebuliser handset with the aim of reducing inhalation times [16].

\section{TREATMENT OF CHRONIC BRONCHIAL INFECTION}

It is important to take into account that bacteria behaves differently in chronic and acute bronchial infection as this has treatment implications. In chronic bronchial infection, bacteria grow on the surface of the epithelium without invading it in a less virulent manner but they can develop persistence mechanisms and cause an inflammatory response. The main persistence mechanisms are the formation of biofilms and hypermutation [17-19]. Within the biofilm, bacteria are in an anaerobic environment which reduces their growth activity and, as a result, the antibacterial effect of the antibiotic. Since antibiotic treatment tends to eliminate the bacteria with more active growth on the biofilm surface, antibiotics have to be administered at high doses and for prolonged periods in order to interfere with the biofilm.

It has long been known that chronic bronchial infection in $\mathrm{CF}$ bronchiectasis should be treated with inhaled antibiotics and very specifically in the case of infections caused by $P$. aeruginosa, which is associated to rapid progression of pulmonary infection in severe cases of $\mathrm{CF}$ [19]. The first antibiotic approved for such use in CF was TSI. Treatment with $300 \mathrm{mg}$ of TSI twice daily in 28-day onoff cycles for a total of 24 weeks was studied in a multicentre, randomised, placebo-controlled trial involving over 500 patients with CF-related bronchiectasis with chronic $P$. aeruginosa bronchial infection. These patients were not receiving antibiotic treatment for the chronic infection. The treatment improved pulmonary function, decreased the density of $P$. aeruginosa in sputum and reduced the risk of exacerbation compared with the placebo [5]. The dry powder tobramycin preparation has been shown to have an efficacy that is comparable to that of TSI in these patients with the same administration regimen [15].

In non-CF bronchiectasis, few studies have been performed with inhaled antibiotics probably as a result of the heterogeneity of the population, the fact that patients have not typically been controlled in specialised units, the generally slower progression of lung damage, and the lack of interest of the pharmaceutical industry, which has had the result that it has repeatedly been not recommended for routine use in these patients [20-23]. However, high bacterial loads in non-CF bronchiectasis are also associated with local and systemic inflammation and a greater risk of exacerbations [24], and chronic P. aeruginosa bronchial infection has been associated with worse quality of life, a greater extent of bronchiectasis, worse lung function [25], and greater mortality $[26,27]$. As with CF bronchiectasis, chronic $P$. aeruginosa bronchial infection may cause more rapid lung function decline if left untreated [28] and can potentially be modified by the use of nebulized antibiotic therapy $[29,30]$. In this regard, both the Spanish and British bronchiectasis guidelines recommend the use of inhaled antibiotics in these patients [31,32].

Four trials have been published evaluating the efficacy of inhaled tobramycin in chronic $P$. aeruginosa bronchial infection patients with stable non-CF bronchiectasis, two of which were conducted by the same group in Catalonia using a continuously administered intravenous formulation [33, $34]$, and two in the USA using TSI $[35,36]$.

Orriols et al. [33] conducted a trial on 17 patients (mean age $62.0 \pm 8.5$ years in the treatment group and $61.4 \pm 10.3$ years in the non-treatment group) who had been treated at least once in the preceding three months with oral ciprofloxacin at doses of $500 \mathrm{mg}$ every 12 hours for 3 weeks due to exacerbation without improvement or recurrence of symptoms. All of the patients received intravenous antibiotic treatment with ceftazidime and tobramycin for 2 weeks. They were subsequently randomly assigned to two groups: 
one group received the same antibiotics that they had received by the intravenous route by inhalation for 12 months (ceftazidime $1 \mathrm{~g}$ every 12 hours in $8 \mathrm{ml}$ of $0.9 \%$ saline solution and tobramycin $100 \mathrm{mg}$ every 12 hours in 8 $\mathrm{ml}$ of $0.9 \%$ saline solution through the System 22 nebuliser and CR60 compressor, Medic-Aid, UK) whereas the other group received only symptomatic treatment. The number and duration of hospitalisations were lower in the patients receiving inhaled antibiotics. Only one patient in the treatment group required hospitalisation, although this patient was admitted four times during the trial period. On the other hand, all but one of the patients who received symptomatic treatment required hospitalisation. No differences were observed regarding the use of oral antibiotics. The eradication of $P$. aeruginosa was not achieved in any patient. Despite the advantages of inhaled antibiotic therapy over leaving the chronic bronchial infection untreated, the administration of two nebulised antibiotics with a considerable amount of solution is timeconsuming for patients, making compliance difficult in clinical practice.

The same group conducted a second trial [34] consisting of a placebo-controlled crossover study of 30 patients (mean age 64.5 years) with an intravenous tobramycin formulation at a higher dose than in the previous trial $(300 \mathrm{mg}$ every 12 hours, TOBRA-GOBENS ${ }^{\circledR} 100 \mathrm{mg} / 2 \mathrm{ml}$, Normon labs, diluted with $0.9 \%$ saline solution to obtain an $8 \mathrm{ml}$ solution) and placebo ( $8 \mathrm{ml}$ of $0.9 \%$ saline) in two 6-month cycles separated by a one-month washout period. The same nebuliser and compressor were used as in the previous trial. At the start of treatment, the patients were also admitted for two weeks of intravenous treatment with two antibiotics (ceftazidime and tobramycin). During treatment with inhaled tobramycin, in addition to fewer and shorter hospitalisations as was found in the previous trial, a decrease in $P$. aeruginosa density in sputum was also observed. The bacterial density in sputum returned to baseline values three months after ending the treatment. No differences were observed with regard to the number of exacerbations, quality of life measured by the St. George Respiratory Questionnaire (SGRQ) or systemic inflammation markers.

Barker et al. [35] conducted a phase II, multicentre, placebo-controlled, double-blind randomised trial in the United States using $300 \mathrm{mg}$ of TSI twice daily administered with the PARI LC PLUS ${ }^{\circledR}$ jet nebuliser and a Pulmo-Aide ${ }^{\circledR}$ compressor (DeVilbiss, Somerset, PA). They used the same methods and system of administration as the Ramsey et al. CF trial [5] published the previous year but applying the treatment over a single 28-day period. Seventy-four patients (mean age $66.6 \pm 13.0$ years in the treatment group and $63.2 \pm 13.5$ years in the placebo group) in stable state with purulent sputum containing at least $10^{4}$ colony-forming units of $P$. aeruginosa per gram who had not been treated with antibiotics in the preceding two weeks were randomly assigned to two groups. One group received TSI and the other received a placebo (1.25 $\mathrm{mg}$ of quinine sulphate in the same excipient). The bacterial density of $P$. aeruginosa in sputum significantly decreased in the patients receiving TSI by the end of the four weeks of treatment when compared with the placebo. This was accompanied by clinical improvement although this was only evaluated by the investigators' subjective assessments of the change in the patients' general medical condition as either "improved" or "not improved". As in the trial in CF patients [5], the greatest reduction in bacterial density was observed after two weeks of treatment and the density was found to have increased again two weeks after stopping the treatment, although the number of colonies remained lower than for the group given the placebo. The extent of bacterial density reduction observed in this trial was greater than that observed in CF patients [5]. The Barker et al. trial, whilst having the largest sample size of the non-CF studies, had considerably fewer patients than Ramsey et al. [5].

In the second American trial with TSI, Scheinberg et al. [36] enrolled 41 patients (mean age 65.2 \pm 13.1 years) with purulent sputum who had received four or more courses of antibiotics for a respiratory condition in the last 12 months in an open-label, multicentre clinical trial. The patients received three treatment cycles with TSI (300 mg/5 ml twice-daily) for 14 days on followed by 14 days off, using the same nebuliser and compressor as in the Barker trial [35]. Upon completion of the three treatment cycles, significant improvements were observed in the symptom severity score as measured by the Patient Symptoms Questionnaire (for 5 symptoms: severity of cough, shortness of breath, sputum production (frequency and severity), fatigue, and wheezing). The greatest reduction in severity and intensity was observed in sputum production. Sputum colour improved in $43.9 \%$ of patients, worsened in $4.9 \%$ and remained unchanged in $51.2 \%$. There was also a significant improvement in quality of life measured by the SGRQ. A greater reduction was found in women than in men with regard to the evaluation of symptoms and quality of life. It is worth noting that $78.6 \%$ of the men had a history of smoking compared with $40.7 \%$ of the women.

Unlike trials in CF, the majority of which have been conducted in young populations, none of the three randomised trials on adult patients with non-CF bronchiectasis showed an improvement in forced expiratory volume in 1 second (FEV1) with the inhaled tobramycin treatment [33-35]. It is likely that change in lung function in these patients is not sufficiently sensitive and should therefore not be an endpoint for analysis. Other variables that are both more sensitive and more specific in terms of reduction in infection should be considered. In fact, almost no other treatment, whether using antibiotics [37-41] or inhaled corticosteroids [42], has managed to improve FEV1 in these patients. Only a phase II trial with a selective neutrophil elastase inhibitor administered to 38 patients over a four-week period has been associated with a significant improvement in FEV1 of $100 \mathrm{ml}$ compared with a placebo [43].

In the four trials, despite the different methods used and the tobramycin formulation administered, beneficial effects to bronchiectasis patients with aetiologies other than $\mathrm{CF}$ were reported. However, none of these studies provided detailed data regarded the aetiology of the bronchiectasis of the enrolled patients. Whilst smoking habits were not specified for either of the Catalonian trials, it is striking that in the American trials, $65 \%$ of the patients who received TSI in the Barker trial [35] and $78.6 \%$ of the men in the Scheinberg trial [36] had a history of smoking, which could involve respiratory diseases caused by smoking in addition 
to the bronchiectasis. The low prevalence of certain aetiologies makes it difficult to conduct trials for each one individually and so data should be extrapolated to other populations given that ultimately it is the bronchial infection that is being treated rather than the cause of bronchiectasis. In any case, more trials are needed to determine the most effective dose and administration protocol in this population, which is generally more elderly and much more heterogeneous than the population with $\mathrm{CF}$. Furthermore, we need to determine whether the most severe cases should receive continued treatment or continue following the current recommendations for TSI in CF patients with on-off periods or whether to add a second inhaled antibiotic during the off period [31].

Although the Murray et al. trial [38] was conducted with inhaled gentamicin and not tobramycin, it is worth mentioning because this drug is probably equivalent to tobramycin in many respects. Sixty-five patients with nonCF bronchiectasis with chronic bronchial infection caused by various pathogenic organisms and at least two exacerbations in the preceding year were randomly allocated to receive twice-daily nebulized gentamicin $(80 \mathrm{mg}$ of gentamicin injectable solution reconstituted for nebulization using $0.9 \%$ saline), or twice-daily nebulized $0.9 \%$ saline $(5 \mathrm{ml})$, with a Porta-Neb Ventstream ${ }^{\circledR}$ nebuliser (Profile Respiratory Systems Ltd, Bognor Regis, West Sussex, UK). Treatment with gentamicin was associated with a reduction in sputum bacterial density, less sputum purulence, greater exercise capacity, improved quality of life (as measured by the SGRQ questionnaire and the Leicester Cough Questionnaire) and fewer exacerbations compared with placebo. After discontinuing the treatment for three months, these improvements were not sustained. Data from this trial suggest that treatment should be prolonged in order to maintain the benefits, that patients with chronic bronchial infection caused by bacteria other than P. aeruginosa also improve with inhaled antibiotics, that higher doses of gentamicin should be studied if they are able to sustain longer-term improvements, and that the results of treatment will probably be better with more efficient nebulisers.

There are no trials comparing inhaled antibiotics in nonCF bronchiectasis. Two trials have compared TSI with new inhaled antibiotics in CF-related bronchiectasis with chronic $P$. aeruginosa bronchial infection $[44,45]$. In the first trial, TSI, at the dose and using the nebuliser that had already proven effective [5] (300 $\mathrm{mg}$ in $5 \mathrm{ml}$ administered with the PARI LC PLUS ${ }^{(}$nebuliser), was compared with colistin (sulphomethate sodium) (Colomycin ${ }^{\circledR}$ injection, Pharmax Ltd, Kent, UK) at a lower dose than what is currently used (1 $\mathrm{mU}$ ) and using a less efficient nebuliser (Ventstream ${ }^{\mathrm{TM}}$ nebuliser, Medic-Aid) for four weeks. The trial concluded that although both antibiotics reduced bacterial density and had equivalent safety profiles, TSI improved the FEV1 after four weeks of treatment while colistin did not. In that trial, $77.4 \%$ of the patients in the group receiving colistin had already been receiving it for at least a month prior to the trial, while only $3.7 \%$ of the patients in the group receiving TSI had received it in the preceding month [44]. In the second trial, aztreonam for inhalation solution (AZLI) (Cayston $^{\circledR}$, Gilead Sciences Inc.) at a dose of $75 \mathrm{mg}$ threetimes daily was compared with TSI (300 mg twice daily). After one treatment cycle with AZLI, the improvement in
FEV1 was higher than with TSI. In both groups, approximately $85 \%$ of the patients had received TSI for at least three months in the preceding year and none had received AZLI [45]. Both trials compared the efficacy of an antibiotic to which the trial population had not been previously submitted versus another antibiotic that had already been widely administered during the previous year. In these cases, the results should be evaluated as the noninferiority rather than the superiority of the antibiotic used in the naive patients versus the antibiotic that had already been used. The TSI [5] and AZLI [45] trials in CF demonstrate that the greatest effect of the inhaled antibiotic on FEV1 occurs within the first 15 days of treatment and that the effect is subsequently sustained during the trial period. Therefore, the effect of the antibiotic on FEV1 improvement is not comparable for the two antibiotics. However, these trials suggest that the rotation or combination of different antibiotics could be a valid therapeutic approach to consider in the treatment of chronic bronchial infection in bronchiectasis patients.

Given the complexity of treating chronic bronchial infection with bacteria growing within biofilm and the challenges of eradicating bacteria, research is being carried out to study the possibility of combining different inhaled antibiotics in order to enhance their antibacterial activity. Fosfomycin is an antibiotic with bactericidal activity against gram-positive and gram-negative bacteria that increases the bactericidal activity of tobramycin. A 4:1 fosfomycin/tobramycin combination showed high bactericidal activity in in vitro and in vivo against bacterial respiratory pathogens seen in the $\mathrm{CF}$ and non-CF bronchiectasis populations such as E. coli, $H$. influenzae, $S$. aureus, Klebsiella spp., and $P$. aeruginosa [46]. In a randomised, placebo-controlled trial in $\mathrm{CF}$ patients with chronic $P$. aeruginosa bronchial infection, this combination at a dose of $160 / 40 \mathrm{mg}$ or $80 / 20 \mathrm{mg}$ twice daily for 28 days was able to maintain the improvements in lung function attained after 28 days of prior treatment with inhaled aztreonam and was well tolerated, although there were fewer adverse effects with the $80 / 20 \mathrm{mg}$ dose [47]. If efficacy is confirmed, this combination would make it possible to substantially reduce the approved dose of tobramycin in TSI in these patients and reduce the potential toxic effects after months or years of treatment. The possibility of combining tobramycin with other antibiotics, including a macrolide [48] or colistin [49], is also being studied.

\section{TREATMENT OF ACUTE BRONCHIAL INFECTIONS- EXACERBATIONS}

The only oral antibiotics available for outpatient use in treating exacerbations in patients with chronic $P$. aeruginosa bronchial infection are ciprofloxacin and levofloxacin. However, these antibiotics may not always be effective and hospitalisation is often required to administer intravenous antibiotic treatment, which increases the overall cost and has a negative impact on quality of life. Bilton et al. [50] conducted a randomised, multicentre, double-blind, placebocontrolled trial to investigate the effect of adding TSI to oral ciprofloxacin for the treatment of exacerbations of non-CF bronchiectasis in 53 adult patients with chronic bronchial infection caused by ciprofloxacin-sensitive $P$. aeruginosa 
(mean age $62 \pm 11.4$ years). Patients were selected during the stable state. At the first exacerbation, they were randomly assigned to two treatment groups. One group received 300 $\mathrm{mg}(5 \mathrm{ml})$ of TSI twice daily with the PARI LC PLUS ${ }^{\circledR}$ jet nebuliser in addition to $750 \mathrm{mg}$ of oral ciprofloxacin twice daily for 14 days. The other group received placebo $(1.25 \mathrm{mg}$ of quinine sulphate [5 ml]) with the same nebuliser and oral ciprofloxacin following the same administration regimen. There was a significantly higher reduction in the sputum density of $P$. aeruginosa during treatment in the group receiving TSI but no significant differences were observed with regard to the reduction seven days after ending the treatment. The greater bacterial reduction was not accompanied by significant differences in clinical improvement in the two groups, which may have been due to the increased frequency of wheezing experienced by the group receiving TSI. Other efficacy variables such as timeto-next-exacerbation and time-to-next-exacerbationrequiring-hospitalization remain to be analysed in future studies.

Only two trials have been conducted to analyse the effect of adding inhaled tobramycin to intravenous antibiotics for treatment of exacerbations in CF-related bronchiectasis patients with chronic $P$. aeruginosa bronchial infection. The first was conducted 30 years ago [51] in 28 patients with CF (age range between 7 and 22 years). It studied an intravenous preparation at a dose of $80 \mathrm{mg} / 8$ hours (Bennet twin jet nebuliser), which was added to intravenous treatment with tobramycin and carbenicillin. The second [52] is more recent and was conducted with TSI $300 \mathrm{mg}$ twice daily added to intravenous tobramycin and colistin. In both trials, the treatment with inhaled tobramycin was associated with a greater reduction in bacterial density in sputum. In the first trial, this decrease was temporary and not accompanied by clinical improvement [51] but in the second trial, with higher doses of TSI and a more effective nebuliser, the treatment was associated with prolonging the time to the next exacerbation requiring hospitalisation without an increase in adverse effects [52].

Although recommendations cannot currently be made regarding the use of inhaled tobramycin for treating exacerbations, future trials should analyse its efficacy in mild exacerbations in patients refractory to treatment with oral ciprofloxacin or in infections caused by resistant $P$. aeruginosa in an attempt to avoid hospitalisations [50].

\section{TREATMENT OF INITIAL COLONISATION WITH PSEUDOMONAS AERUGINOSA}

The eradication of $P$. aeruginosa in chronic bronchial infection is very difficult to achieve, particularly in CFrelated bronchiectasis. There is a greater chance of success, however, during the period of initial colonisation when $P$. aeruginosa are more sensitive to antibiotics, they are still unable to form biofilms, and the bacterial density is low. Although several eradication protocols have been studied in CF patients with promising results, including inhaled tobramycin alone and in combination with oral ciprofloxacin, the most effective protocol has yet to be determined [53].
We have no knowledge of trials attempting to eradicate the initial $P$. aeruginosa colonisation in non-CF bronchiectasis with an inhaled antibiotic. Nevertheless, both Spanish and British guidelines recommend early treatment of initial $P$. aeruginosa colonisation with an inhaled antibiotic if the attempt to eradicate it with oral ciprofloxacin fails [31, 32].

\section{ADVERSE EFFECTS}

Inhaled tobramycin is generally well tolerated. Systemic absorption in bronchiectasis patients is low. The mean serum concentration in patients treated with $300 \mathrm{mg}$ of TSI, measured 30 and 60 minutes after administration during stable phase, was $0.54 \mu \mathrm{g} / \mathrm{ml}$ and ranged from the lower limit of detection $(0.18 \mu \mathrm{g} / \mathrm{ml})$ to $2.64 \mu \mathrm{g} / \mathrm{ml}$ [35]. The serum concentration during exacerbations 60 minutes after administration was $1.14 \mu \mathrm{g} / \mathrm{ml}$ (SD: $0.68 \mu \mathrm{g} / \mathrm{ml}$, range: 0.29 to $2.34 \mu \mathrm{g} / \mathrm{ml}$ ) [50]. These data are comparable to the concentrations in $\mathrm{CF}$ patients [5]. Trials in bronchiectasis patients found no renal or vestibular toxicity [33-35, 50], and showed only a decrease in creatinine clearance in one trial [36] and transitory mild or moderate tinnitus with no audiometric changes in another [34]. However, patients with impaired kidney function or audiometric disorders are excluded from the majority of trials [33, 34, 50] and so patients with these conditions should be treated with particular caution [8]. Sporadic cases of systemic toxicity have been reported both in CF patients [54] and patients with conditions other than bronchiectasis in whom there may be greater systemic absorption of tobramycin $[55,56]$.

Compared to the CF trials [5], adverse respiratory effect reports are more common in bronchiectasis secondary to other aetiologies [33-36, 50]. Bronchospasm was initially associated with hypertonic solutions and the presence of sulphites or other additives in intravenous preparations [10]. In the Orriols et al. trial, the inhaled treatment with an unspecified intravenous formulation of tobramycin was discontinued in one out of 17 patients due to bronchospasm despite intensive bronchodilator treatment being given; the patient had a previous history of bronchial hyperreactivity [33]. In the Drobnic et al. trial, $10 \%$ of the patients had bronchospasm, which forced them to discontinue treatment. One patient out of 20 experienced shortness of breath and wheezing during the first month of treatment and required bronchodilator treatment until the end of the trial. The intravenous formulation used in this study was TOBRAGOBENS $^{\circledR}$ [34]. The rate of adverse respiratory effects with TSI was higher than with placebo in the Barker et al. trial (4$16 \%$ of the patients had cough, shortness of breath or wheezing associated with TSI) [35]. In the Scheinberg et al. trial, $26.8 \%$ of patients had cough, $17.1 \%$ dyspnoea and $17.1 \%$ wheezing, which were associated with TSI. A total of $22 \%$ of patients were withdrawn from the trial due to side effects [36]. Cough and wheezing were identified when treating exacerbations, although these symptoms were not suspected on measuring changes in FEV1 at 30 minutes post-dose on the first day of treatment [50]. The greatest differences between patients with $\mathrm{CF}$ and those with bronchiectasis secondary to other aetiologies, which could explain the greater incidence of respiratory side effects among the non-CF patients, are the greater age in the non-CF 
patients (66.6 years vs 20.8 years in CF) and a greater history of smoking (65\% of patients in the Barker trial [35] and $53.7 \%$ in the Scheinberg trial [36]). It is advisable that the first administration of treatment should be given in hospital in order to monitor the possibility of any respiratory adverse effects [32].

A decrease in the susceptibility of $P$. aeruginosa to antibiotics and the emergence of multi-resistant pathogens are major concerns in the use of long-term inhaled antibiotic therapy. Clinical trials with inhaled tobramycin have not found a significant increase in the resistance of $P$. aeruginosa compared to placebo or no treatment [33-35]. However, in the Barker et al. trial, four of the patients receiving TSI had resistant isolates of $P$. aeruginosa based on the current breakpoints for parenteral tobramycin $(\geq 16$ $\mu \mathrm{g} / \mathrm{ml})[35]$.

Given the high bronchial concentration reached by inhaled antibiotics, much higher breakpoints have been proposed for inhaled tobramycin (susceptible $\leq 64 \mu \mathrm{g} / \mathrm{ml}$ and resistent $\geq 128 \mu / \mathrm{ml})[57]$.

\section{SUMMARY}

To summarise, trials conducted with inhaled tobramycin in chronic $P$. aeruginosa bronchial infection patients with non-CF bronchiectasis have shown clinical improvement and reduction in bacterial density. Further trials are needed to determine the most effective dose and administration protocol in these patients. Based on the current evidence, recommendations cannot be made regarding the use of inhaled tobramycin to treat exacerbations. Although no systemic toxicity has been reported with this treatment, patients with known kidney disease or ear disorders should be treated with caution. Moreover, adverse respiratory effects are reported to be more common in non-CF patients that in CF patients, who are generally non-smokers and younger. Research is ongoing into the possibility of combining tobramycin with other antibiotics to increase its antibacterial activity.

\section{CONFLICT OF INTEREST}

Chiesi sponsored the review but had no involvement in the contents.

Dr. de Gracia has received personal fees for participating on advisory boards from Gilead Sciences, Novartis and Chiesi; grants from Gilead Sciences and Novartis; and fees for lectures from Praxis, Novartis and Chiesi.

Dr. Vendrell has received fees for lectures from Praxis, Novartis and Chiesi and a grant from Praxis.

\section{ACKNOWLEDGEMENTS}

The authors thank Andrew Hughes for the English correction.

\section{REFERENCES}

[1] Olsen AM. Streptomycin aerosol in the treatment of chronic bronchiectasis; preliminary report. Proc Staff Meet Mayo Clin 1946; $21: 53$.
[2] Wall MA, Terry AB, Eisenberg J, McNamara M, Cohen R. Inhaled antibiotics in cystic fibrosis. Lancet 1983; 1: 1325.

[3] Carswell F, Ward C, Cook DA, Speller DCE. A controlled trial of nebulized aminoglycoside and oral flucloxacillin versus placebo in the outpatient management of children with cystic fibrosis. Br J Dis Chest 1987; $81: 356-60$.

[4] MacLusky IB, Gold R, Corey M, Levison H. Long-term effects of inhaled tobramycin in patients with cystic fibrosis colonized with Pseudomonas aeruginosa. Pediatr Pulmonol 1989; 7: 42-48.

[5] Ramsey BW, Pepe MS, Quan JM, et al. Intermittent administration of inhaled tobramycin in patients with cystic fibrosis: Cystic Fibrosis Inhaled Tobramycin Study Group. N Engl J Med 1999; 340: $23-30$.

[6] Ryan G, Mukhopadhyay S, Singh M. Nebulised antipseudomonal antibiotics for cystic fibrosis (Cochrane Review). In: The Cochrane Library, Issue 3, Oxford: Update Software 2000.

[7] Cheer SM, Waugh J, Noble S. Inhaled tobramycin (TOBI). A review of its use in the management of Pseudomonas aeruginosa infections in patients with cystic fibrosis. Drugs 2003; 63: 2501-20.

[8] Singh MP. Tobramycin (Cystic Fibrosis Foundation/PathoGenesis). Curr Opin Investig Drugs 2001; 2: 755-65.

[9] Chuchalin A, Amelina E, Bianco F. Tobramycin for inhalation in cystic fibrosis: Beyond respiratory improvements. Pulm Pharmacol Ther. 2009; 22: 526-32.

[10] Alothman GA, Alsaadi MM, Ho BL, et al. Evaluation of bronchial constriction in children with cystic fibrosis after inhaling two different preparations of tobramycin. Chest 2002; 122: 930-4.

[11] Standaert TA, Vandevanter D, Ramsey BW, et al. The choice of compressor effects the aerosol parameters and the delivery of tobramycin from a single model nebulizer. J Aerosol Med 2000; 13: $147-53$.

[12] Lenney W, Edenborough F, Kho P, Kovarik JM. Lung deposition of inhaled tobramycin with eFlow rapid/LC Plus jet nebuliser in healthy and cystic fibrosis subjects. J Cyst Fibros 2011; 10: 9-14.

[13] Poli G, Acerbi D, Pennini R, et al. Clinical pharmacology study of Bramitob, a tobramycin solution for nebulization, in comparison with Tobi. Paediatr Drugs 2007; 9(Suppl 1): 3-9.

[14] Mazurek H, Chiron R, Kucerova T, et al. Long-term efficacy and safety of aerosolized tobramycin $300 \mathrm{mg} / 4 \mathrm{ml}$ in cystic fibrosis. Pediatr Pulmonol 2014; 49: 1076-89.

[15] Konstan MW, Flume PA, Kappler M, et al. Safety, efficacy and convenience of tobramycin inhalation powder in cystic fibrosis patients: The EAGER trial. J Cyst Fibros 2011; 10: 54-61.

[16] Sands D, Sapiejka E, Gąszczyk G, Mazurek H; for the T100 Study Group. Comparison of two tobramycin nebuliser solutions: Pharmacokinetic, efficacy and safety profiles of T100 and TNS. J Cyst Fibros 2014; 13: 653-60.

[17] Maciá MD, Blanquer D, Togores B, Sauleda J, Pérez JL, Oliver A. Hypermutation is a key factor in development of multipleantimicrobial resistance in Pseudomonas aeruginosa strains causing chronic lung infections. Antimicrob Agents Chemother. 2005; 49: 3382-6.

[18] Chalmers JD, Hill AT. Mechanisms of immune dysfunction and bacterial persistence in non-cystic fibrosis bronchiectasis. Mol Immunol 2013;55:27-34.

[19] Cantón R, Cobos N, de Gracia J, et al. Antimicrobial therapy for pulmonary pathogenic colonisation and infection by Pseudomonas aeruginosa in cystic fibrosis patients. Clin Microbiol Infect 2005; 11: 690-703.

[20] Woodhead M, Blasi F, Ewig S, et al. Guidelines for the management of adult lower respiratory tract infections. Eur Respir J 2005; 26: 1138-80.

[21] Rosen MJ. Chronic cough due to bronchiectasis: ACCP evidencebased clinical practice guidelines. Chest 2006; 129(Suppl 1): 122S$131 \mathrm{~S}$.

[22] Woodhead M, Blasi F, Ewig S, et al. Guidelines for the management of adult lower respiratory tract infections--full version. Clin Microbiol Infect. 2011;17 (Suppl 6): E1-59.

[23] Le J, Ashley ED, Neuhauser MM, et al. Consensus summary of aerosolized antimicrobial agents: application of guideline criteria. Insights from the Society of Infectious Diseases Pharmacists. Pharmacotherapy 2010; 30: 562-84.

[24] Chalmers JD, Smith MP, McHugh BJ, Doherty C, Govan JR, Hill AT. Short- and long-term antibiotic treatment reduces airway and systemic inflammation in non-cystic fibrosis bronchiectasis. Am J Respir Crit Care Med 2012; 186: 657-65. 
[25] Wilson CB, Jones PW, O'Leary CJ, Hansell DM, Cole PJ, Wilson R. Effect of sputum bacteriology on the quality of life of patients with bronchiectasis. Eur Respir J 1997; 10: 1754-60.

[26] Martínez-García MÁ, de Gracia J, Vendrell Relat M, et al. Multidimensional approach to non-cystic fibrosis bronchiectasis: the FACED score. Eur Respir J 2014; 43: 1357-67.

[27] Chalmers JD, Goeminne P, Aliberti S, et al. The bronchiectasis severity index. An international derivation and validation study. Am J Respir Crit Care Med 2014; 189: 576-85.

[28] Martinez-Garcia MA, Soler-Cataluña JJ, Perpiñá-Tordera M, Román-Sánchez P, Soriano J. Factors associated with lung function decline in adult patients with stable non-cystic fibrosis bronchiectasis. Chest 2007; 132: 1565-72.

[29] Davies G, Wells AU, Doffman S, et al. The effect of pseudomonas aeruginosa on pulmonary function in patients with bronchiectasis. Eur Respir J 2006; 28: 974-79.

[30] Bilton D. Update on non-cystic fibrosis bronchiectasis. Curr Opin Pulm Med 2008; 14: 595-9.

[31] Vendrell M, de Gracia J, Olveira C, et al. Diagnosis and treatment of bronchiectasis. Spanish Society of Pneumology and Thoracic Surgery. Arch Bronconeumol. 2008; 44: 629-40.

[32] Pasteur MC, Bilton D, Hill AT; British Thoracic Society Bronchiectasis non-CF Guideline Group. British Thoracic Society guideline for non-CF bronchiectasis. Thorax 2010; 65(Suppl 1): i158.

[33] Orriols R, Roig J, Ferrer J, et al. Inhaled antibiotic therapy in noncystic fibrosis patients with bronchiectasis and chronic bronchial infection by Pseudomonas aeruginosa. Respir Med 1999; 93: 47680 .

[34] Drobnic ME, Suñé P, Montoro JB, Ferrer A, Orriols R. Inhaled tobramycin in non-cystic fibrosis patients with bronchiectasis and chronic bronchial infection with Pseudomonas aeruginosa. Ann Pharmacother 2005; 39: 39-44.

[35] Barker AF, Couch L, Fiel SB, et al. Tobramycin solution for inhalation reduces sputum Pseudomonas aeruginosa density in bronchiectasis. Am J Respir Crit Care Med 2000; 162: 481-85.

[36] Scheinberg P, Shore E. A pilot study of the safety and efficacy of tobramycin solution for inhalation in patients with severe bronchiectasis. Chest 2005; 127: 1420-6.

[37] Haworth CS, Foweraker JE, Wilkinson P, Kenyon RF, Bilton D. Inhaled colistin in patients with bronchiectasis and chronic pseudomonas aeruginosa infection. Am J Respir Crit Care Med 2014; 189: 975-82.

[38] Murray MP, Govan JR, Doherty CJ, et al. A randomized controlled trial of nebulized gentamicin in non-cystic fibrosis bronchiectasis. Am J Respir Crit Care Med 2011; 183: 491-9.

[39] Wilson R, Welte T, Polverino E, et al. Ciprofloxacin dry powder for inhalation in non-cystic fibrosis bronchiectasis: a phase II randomised study. Eur Respir J 2013; 41: 1107-15.

[40] Serisier DJ, Bilton D, De Soyza A, et al. Inhaled, dual release liposomal ciprofloxacin in non-cystic fibrosis bronchiectasis (ORBIT-2): a randomised, double-blind, placebo-controlled trial. Thorax 2013; 68: 812-7.

[41] Wong C, Jayaram L, Karalus N, et al. Azithromycin for prevention of exacerbations in non-cystic fibrosis bronchiectasis
(EMBRACE): a randomised, double-blind, placebo-controlled trial. Lancet 2012; 380: 660-7.

[42] Tsang $\mathrm{KW}$, Tan $\mathrm{KC}$, Ho PL, et al. Inhaled fluticasone in bronchiectasis: a 12 month study. Thorax 2005; 60: 239-43.

[43] Stockley R, De Soyza A, Gunawardena K, et al. Phase II study of a neutrophil elastase inhibitor (AZD9668) in patients with bronchiectasis. Respir Med 2013; 107: 524-33.

[44] Hodson ME, Gallagher CG, Govan JR. A randomised clinical trial of nebulised tobramycin or colistin in cystic fibrosis. Eur Respir J 2002; 20: 658-64.

[45] Assael BM, Pressler T, Bilton D, et al. Inhaled aztreonam lysine vs inhaled tobramycin in cystic fibrosis: A comparative efficacy trial. J Cyst Fibros 2013; 2: 130-40.

[46] MacLeod DL, Barker LM, Sutherland JL, et al. Antibacterial activities of a fosfomycin/tobramycin combination: a novel inhaled antibiotic for bronchiectasis. J Antimicrob Chemother 2009; 64: 829-36.

[47] Trapnell BC, McColley SA, Kissner DG, et al. Fosfomycin/tobramycin for inhalation in patients with cystic fibrosis with Pseudomonas airway infection. Am J Respir Crit Care Med 2012; 185: 171-8.

[48] Tré-Hardy M, Nagant C, El Manssouri N, et al. Efficacy of the combination of tobramycin and a macrolide in an in vitro Pseudomonas aeruginosa mature biofilm model. Antimicrob Agents Chemother 2010; 54: 4409-15.

[49] Herrmann G, Yang L, Wu H, et al. Colistin-tobramycin combinations are superior to monotherapy concerning the killing of biofilm Pseudomonas aeruginosa. J Infect Dis 2010; 202: 1585-92.

[50] Bilton D, Henig N, Morrisey B, Gotfried M. Addition of inhaled tobramycin to ciprofloxacin for acute exacerbations of Pseudomonas aeruginosa infection in adult bronchiectasis. Chest 2006; 130: 1503-10.

[51] Stephens D, Garey N, Isles A, Levison H, Gold R. Efficacy of inhaled tobramycin in the treatment of pulmonary exacerbations in children with cystic fibrosis. Pediatr Infect Dis 1983; 2: 209-11.

[52] Al-Aloul M, Nazareth D, Walshaw M. Nebulized Tobramycin in the Treatment of Adult CF Pulmonary Exacerbations. J Aerosol Med Pulm Drug Deliv 2014; 27: 299-305.

[53] Döring G, Hoiby N; Consensus Study Group. Early intervention and prevention of lung disease in cystic fibrosis: a European consensus. J Cyst Fibros 2004; 3: 67-91.

[54] Hoffmann IM, Rubin BK, Iskandar SS, Schechter MS, Nagaraj SK, Bitzan MM. Acute renal failure in cystic fibrosis: association with inhaled tobramycin therapy. Pediatr Pulmonol 2002; 34: 375-7.

[55] Ahya VN, Doyle AM, Mendez JD, et al. Renal and vestibular toxicity due to inhaled tobramycin in a lung transplant recipient. J Heart Lung Transplant 2005; 24: 932-5.

[56] Izquierdo MJ, Gomez-Alamillo C, Ortiz F, et al. Acute renal failure associated with use of inhaled tobramycin for treatment of chronic airway colonization with Pseudomonas aeruginosa. Clin Nephrol 2006; 66: 464-7.

[57] Morosini MI, García-Castillo M, Loza E, Pérez-Vázquez M, Baquero F, Cantón R. Breakpoints for predicting Pseudomonas aeruginosa susceptibility to inhaled tobramycin in cystic fibrosis patients: use of high-range Etest strips. J Clin Microbiol 2005; 43: 4480-5.

(C) Vendrell et al.; Licensee Bentham Open.

This is an open access article licensed under the terms of the Creative Commons Attribution Non-Commercial License (http://creativecommons.org/licenses/by-nc/3.0/) which permits unrestricted, non-commercial use, distribution and reproduction in any medium, provided the work is properly cited. 